Itinéraires Itinéraires

Littérature, textes, cultures

\title{
Inscrire son corps dans le texte : comment s'hybrigraphier?
}

Bernard Andrieu

\section{(2) OpenEdition}

1 Journals

Édition électronique

URL : http://journals.openedition.org/itineraires/386

DOI : 10.4000/itineraires.386

ISSN : 2427-920X

Éditeur

Pléiade

\section{Édition imprimée}

Date de publication : 1 mai 2009

Pagination : 161-179

ISBN : 978-2-296-08444-5

ISSN : 2100-1340

\section{Référence électronique}

Bernard Andrieu, «Inscrire son corps dans le texte : comment s'hybrigraphier? », Itinéraires [En ligne], 2009-1 | 2009, mis en ligne le 13 juin 2014, consulté le 02 juillet 2020. URL : http://

journals.openedition.org/itineraires/386 ; DOI : https://doi.org/10.4000/itineraires.386

Ce document a été généré automatiquement le 2 juillet 2020

\section{cc) (i) () $\Theta$}

Itinéraires est mis à disposition selon les termes de la licence Creative Commons Attribution - Pas

d'Utilisation Commerciale - Pas de Modification 4.0 International. 


\title{
Inscrire son corps dans le texte : comment s'hybrigraphier?
}

\author{
Bernard Andrieu
}

\section{Introduction}

1 La corpographèse écrit et inscrit sur les corps des discours, des identités et des marques dans le but de constituer une identité plus ressemblante que celle qui est fournie par notre hérédité naturelle. Les représentations de ces marques varient avec les cultures. Dans les sociétés dites primitives, elles peuvent avoir un sens d'appartenance collective de l'individu comme dans les rituels d'initiation ou de passage : la marque s'effectue à un moment donné de l'existence individuelle, comme le passage de l'enfance à l'âge adulte, réduisant ainsi la période de l'adolescence afin de préparer l'accès à la guerre, à la chasse et à la sexualité. Dans les sociétés contemporaines, les marques sur le corps ont perdu cette signification collective d'appartenance à un monde symbolique, à cause de notre désenchantement du monde et de notre croyance en l'individualisme; mais, comme l'expriment des revues comme Body Art à propos du tatouage et des pratiques sado-masochistes du piercing, l'individu occidental cherche à se faire reconnaître à l'intérieur d'un groupe par une marque singulière, liée à son histoire personnelle. Face à cette écriture de soi, les moralisateurs du corps estiment que le corps est un adversaire pour le sujet qui devrait se battre contre lui, pour lui imposer une forme et une norme (voir Marzano 2007). Sans doute assiste-t-on aujourd'hui à une tentative de réappropriation de la marque sur le corps comme mode d'inscription individuelle du sujet dans la société (la marque comme moyen de se faire re-marquer, et comme modalité de la stigmatisation dans le corps social).

2 Mais, si le biopouvoir, désormais électronique, marque la pratique corporelle, nous voudrions aller au-delà de cette constatation et la caractériser moins comme une corpographèse subie que comme une hybrigraphèse vécue et revendiquée pour définir subjectivement ses propres normes de sujet incarné. En décidant de ses expériences tactiles, le corps $\mathrm{du} \mathrm{xxI}^{\mathrm{e}}$ siècle construit une identité subjective dont l'intégration 
politique et la reconnaissance juridique ne concernent plus seulement les gays, les lesbiennes, les personnes changeant de sexe comme les transgenres et autres queers. Les greffés, les implantés, les OGM et les créatures hybrides ne changent pas seulement d'apparence pour se maintenir dans le standard érotique de la beauté normative. Ils inscrivent un nouveau corps dans leur chair.

Ces modifications sensorielles tendent à harmoniser l'image du corps, le schéma corporel, l'estime de soi et le regard d'autrui dans un corps désormais écrit par soi. L'inscription n'est plus un obstacle à la découverte du soi corporel, mais une mise en écriture du dehors par le dedans lesbien, homosexuel, sensuel ou travesti. La difficulté est de se dégenrer (de quitter son identité sexuelle pour prendre un autre genre) sans perdre son identité en faisant des expériences tactiles.

Christophe Prochasson comme un empire des émotions : "Comment écrire sur ce que l'on connait directement, voire sur soi, en renonçant à un style qui apparenterait l'analyse au simple récit d'une expérience personnelle, au pire à un règlement de comptes?» (Prochasson 2008: 65). L'histoire des sensibilités est certes devenue une clinique de l'écriture (Artières 1995: 82-95) comme prose du corps, mais les tatouages de prisonniers, les bracelets de parchemin (Farge 2003 : 17) sont autant d'écrits de soi sur soi sans lesquels toute inscription identitaire disparaîtrait.

En signant sur le corps l'histoire du sujet, l'expérience corporelle transforme la marque en référent d'individuation. Le récit des corps (Farge 2007 : 173) va au-delà du récit de soi en incarnant une expérience soit à même la peau tannée, blessée ou modifiée, soit dans la chair intime, laquelle échappe au langage corporel qui tente de la décrypter. Le sujet incarnant ses marques assume son identité moins comme une revendication contestataire que comme une action remarquable d'existence.

6 La déconstruction des sciences du corps a été entamée par les philosophes du soupçon dans leur étiologie subjective. La déconstruction de l'essentialisme entraîne-t-elle pour autant la fin de toute référence à un naturalisme ? L'excès de la naturalisation trouve son double dans la rigueur de la dénaturalisation. S'appuyant sur une critique du gender de la génétique et de l'évolutionnisme (voir Haraway 1991), la dénaturalisation présuppose à juste titre que l'opposition nature/nurture n'a plus de sens, ou plutôt révèle une orientation masculine de l'idéologie scientiste.

\section{Du biopouvoir au gouvernement de soi}

\section{Corpographèse du féminin : un processus masculin ?}

7 Selon Emily Martin, l'étude des métaphores du corps place la femme dans son corps (règles, accouchement) à travers le langage, les images, les dispositifs sanitaires et les lieux de travail :

Bien que les femmes résistent à des procédures médicales spécifiques comme la césarienne ou l'anesthésie pendant l'accouchement, elles semblent ne pas pouvoir résister aux présupposés qui sous-tendent ces procédures : à savoir que le sujet et le corps sont séparés, que les contractions sont involontaires, que la naissance est production.

8 À travers les pratiques corporelles réelles vécues par les femmes se découvre la construction du corps anatomique, sexuel et social par le moyen du savoir masculin 
(voir Jardine 1985). The Woman in the Body (et non the body in the woman), est une analyse culturelle de la reproduction. La femme a été placée dans la métaphore corporelle par le biais d'une science médicale (voir Martin 1987b) à travers les représentations et les classifications culturelles. Le langage et l'imagerie culturelle définissent les représentations de la naissance, de la contraception et même du corps de la femme, à l'intérieur même des désignations scientifiques.

Nelly Oudshoor décrit l'émergence de l'endocrinologie sexuelle à travers les réseaux entre gynécologie et industries pharmaceutiques qui instrumentalisent le corps même de la femme. Dépassant l'analyse du strict laboratoire et de ses idéologies qui dirigeraient les acteurs de la science, elle montre que «non seulement le laboratoire reflète les préjugés de la société liés au genre, mais il est précisément le lieu où le genre se construit et se métamorphose ${ }^{2}$ ». Le corps devient lui-même, avec l'institution de la recherche biotechnologique, un matériau avec un statut construit « de phénomène naturel universel » (Oudshoor $2000:$ 42).

L'accentuation ou l'élimination de la séparation des genres structure la perception des organes et de l'activité sexuels et la justification de la division sexuelle des tâches sociales en utilisant la naturalisation comme alibi idéologique. Dans la perspective d'un féminisme de la subversion (voir Butler 1990/2005), le genre se situe dans la subversion des styles corporels que nous accomplissons de manière performative lors de la répétition et du rituel des postures et des actes sociaux que chaque sexe accomplit : « Il devient possible de montrer que ce que nous pensons être une propriété "interne" à nous-même doit être mis sur le compte de ce que nous attendons et produisons à travers certains actes corporels.» (Butler 2005 : 36) Le corps est entre sexe et genre, posant ainsi la question de la ligne de démarcation (voir Kraus 2005 : 57) entre la nature et le social afin d'éviter tant une dénaturalisation par un constructivisme social qu'une réduction par un naturalisme revivifié par les travaux sur la bio-construction développementale.

\section{Vers l'engagement corporel du sujet}

11 Ces pratiques de bioconstruction du corps (voir Travaillot 1998) définissent une esthétique de l'existence et un style corporel dans la tradition analysée par le dernier Michel Foucault. Dans leur commentaire qui assimile «le problème du gouvernement du corps et de ses avatars de l'Histoire de la folie jusqu'au Souci de soi en passant par Surveiller et Punir » (Fassin et Memmi $2004:$ 20-22), Didier Fassin et Dominique Memmi démontrent combien la substitution de la notion de gouvernement à celle de biopouvoir participe bien du basculement vers une herméneutique du sujet.

Dans le cours de 1981-1982, L'Herméneutique du sujet, Foucault utilise les termes de technologie de soi, techniques de soi et tekhnê tou biou («l'art, la procédure réfléchie d'existence, la technique de vie », Foucault $2001: 171$ ) pour caractériser ces techniques, non disciplinaires car décidées par le sujet pour lui-même. L'exercice sur soi est une écriture spirituelle (Benatouil 2003: 29) et un exercice corporel: à l'inverse de la toupie, qui serait plutôt le modèle de la biopolitique, laquelle «tourne sur soi à la sollicitation et sous l'impulsion d'un mouvement extérieur", la sagesse refuse l'impulsion par le mouvement involontaire, car « il faudra chercher au centre de soimême le point auquel on se fixera et par rapport auquel on restera immobile" (Foucault 2001: 199). Les techniques de soi, comme l'ascèse, les régimes des 
abstinences-exploits, des abstinences-épreuves, méditations de la mort, etc., sont des instruments au service de la tekhnê ton biou plutôt que des finalités propres ${ }^{3}$.

Dans sa leçon du 5 janvier 1983 sur le gouvernement de soi et des autres,

il s'agissait d'analyser l'axe de constitution du mode d'être du sujet. Et là le déplacement a consisté en ceci que, plutôt que de se référer à une théorie du sujet, il [lui] a[vait] semblé qu'il fallait essayer d'analyser les différentes formes par lesquelles l'individu est amené à se constituer lui-même comme sujet... voir comment et à travers quelles formes concrètes de rapport à soi, l'individu avait été appelé à se constituer comme sujet. (Foucault $2003: 6$ )

Là où les technologies non disciplinaires gouvernent par l'insertion de l'individu dans une population, l'herméneutique d'un sujet a le souci de soi :

c'est le développement d'un art de l'existence qui gravite autour de la question du soi, de sa dépendance et de son indépendance, de sa forme universelle et du lien qu'il peut et doit établir aux autres, des procédures par lesquelles il exerce son contrôle sur lui-même et de la manière dont il peut établir la pleine souveraineté sur soi. (Foucault 1984b : 273)

Si l'on pense en termes de sujet incarné et plus seulement d'individu corporel, l'engagement corporel (voir Soulié et Corneloup 2007) est plus intense, au point que rechercher dans la marque obtenue par l'expérience extrême et risquée devient un mode de valorisation. La fabrique de son corps (Meidani 2007 : 27) place le sujet incarné à la fois devant l'obligation sociale de sculpter ses formes pour se conformer à la norme sociale et devant celle de s'y rechercher des modes de satisfaction narcissiques.

Ce passage de la corpographèse du biopouvoir à un engagement corporel du sujet suppose une inscription corporelle (Varela, Thompson et Rosch 1993 : 210) dans une énaction, c'est-à-dire une action incarnant le sujet car le sujet percevant s'inscrit dans son corps en la qualifiant. Comment trouver un dispositif d'écriture qui montre le sujet s'inscrivant dans l'acte même d'écrire le corps? Le récit autobiographique, réduisant l'inscription à un processus de légitimation du récit par l'auteur, n'y suffit pas.

\section{De la corpographèse à l'hybrigraphèse}

17 L'hybrigraphèse est au contraire une technique corporelle pour inscrire la marque subjective en y montrant la modification du vécu, du schéma et de l'image corporels. S'hybrigraphier est une action-writing ${ }^{4}$ que nous étudierons sur trois plans, épistémologique, esthésiologique et biotechnologique, lesquels constituent trois degrés dans l'émergence d'un sujet réellement incarné et une hybridation de plus en plus incorporée :

- l'inscription épistémologique d'une autre culture pose le problème d'une incorporation par hybridation comme dans l'hybrigraphèse de l'autre de Maurice Leenhardt qui inscrit le corps dans le monde mélanésien, où il était jusque-là inconnu. L'hybrigraphèse révèle la frontière épistémologique entre les discours ;

- l'inscription érographique renouvelle l'hybridation sexuelle par la dégendérisation des rapports au gode et autres techniques sensorielles: le queer n'en reste pas à défaire le genre : il donne les moyens conceptuels et pratiques de renouveler les modes d'inscription de la sensorialité ;

- l'inscription biocyborg, dans la perspective de Donna Haraway, implante la technique dans le corps même par des prothèses et des dispositifs interactifs avec l'environnement vécu. 


\section{L'hybrigraphèse de l'autre}

18 Maurice Leenhardt (1878-1954) étudie, dans son livre Do Kamo. La personne et le mythe dans le monde mélanésien (1947) la représentation «à la fois empirique, esthétique et mythique» du corps (Leenhardt 1947: 54). Le Canaque ignore la profondeur, développant une vision en deux dimensions, produisant à la fois une expression verbale et une expression esthétique pour la désignation dans le langage des parties du corps. Le corps du chercheur ne peut plus clairement distinguer le corps comme unité isolable dans la langue mélanésienne; il doit renoncer à la catégorisation occidentale, car le vocable Karo « convient à nombre d'expressions qui permettent dans l'ensemble d'en saisir le sens propre» (Leenhardt 1947: 68). Comme «notion technique » (Leenhardt 1947: 69), le vocable Karo ajoute une signification à un autre vocable, comme pour Karo gi, le corps de la hache, c'est-à-dire le manche.

«Comment aller au-delà de cette première notion et la transposer pour que le Mélanésien se sente appuyé soi-même sur son propre corps ? " : Leenhardt démontre combien la transposition de la notion technique à la notion de centre propre du corps personnel serait une interprétation. Parler de corps du chercheur et du corps lui-même suppose que l'on puisse culturellement classer l'identité substantielle, séparer en une représentation le corps propre, se distinguer, en tant qu'individu, du monde. Or

la question est vaine, parce que le Canaque ne se la pose pas. Il ne peut pas y songer, puisqu'il ne s'est pas dégagé de lui-même. Qu'on y songe bien, une représentation, pour être entière, pose nécessairement l'existence d'un contenu. Et le contenu propre du corps reste pour le Mélanésien, indéfinissable. (Leenhardt 1947 : 69)

Le corps du chercheur révèle la constitution du corps comme objet pour un sujet réflexif à l'intérieur d'une culture l'ayant unifié, catégorisé et identifié au moi d'un sujet autonome par rapport au monde.

Or dans le monde mélanésien, le monde viscéral est pareil au monde végétal, «corps organique, corps psychologique s'enrobent l'un dans l'autre» (Leenhardt 1947: 69), alors que nous avons en Occident, comme nous l'avons montré ailleurs, séparé corps psychologique et corps organique en les assignant, sauf dans la perspective holistique, à des disciplines contradictoires (voir Andrieu 1993). La division du sujet contemporain repose sur la dispersion épistémologique du corps. La question de la participation ne peut apparaitre que dans un contexte de dispersion ontologique où le sujet est à la recherche de son corps perdu et éclaté dans différents domaines en raison de l'opposition entre le monde et le corps.

Dans la mesure où nous nous sommes dégagés du monde par (ce que nous croyons être) la maitrise sur lui au moyen de la science et de la technique, cette constitution de la subjectivité repose sur la notion de profondeur du moi (jusqu'à l'inconscient), si étrangère au monde à deux dimensions des Mélanésiens: "Il faudrait qu'à la représentation plastique d'un corps jouant le rôle de soutien, le Mélanésien ajoute celle d'un moi lié à l'organisme. Mais c'est lui demander l'impossible. » (Leenhardt 1947 : 69) Dès lors que nous lions l'organisme au moi, nous attribuons au chercheur son corps comme une cause d'influence de ses représentations internes, faute de pouvoir entièrement comprendre le monde culturel étudié. Pour le Mélanésien il y a un "abîme" (Leenhardt 1947: 70) entre le moment où le Canaque nomme son corps et celui où il sait que son corps et lui ne font qu'un. Ce qui caractérise notre occidentalité 
c'est d'avoir franchi cet abîme au point même de faire du langage de l'intériorité le principe de la projection sur le monde de nos jugements et de nos croyances.

L'externalisation de son corps dans le monde est impossible pour le Mélanésien : « Et le Mélanésien ignorant que ce corps est un élément dont il est, lui, le possesseur, se trouve du même coup dans l'impossibilité de le dégager. Il ne peut l'extérioriser hors de son milieu naturel, social, mythique. Il ne peut l'isoler. Il ne peut voir en lui l'un des éléments de l'individu.» (Leenhardt 1947 : 70) L'externalisation de notre corps n'est possible que si nous pouvons délimiter notre corps dans une unité et une identité individuelle susceptible d'être séparée du monde : en délimitant comme in-dividu son corps, le sujet occidental a perdu le soutien du Karo dans Kamo, le qui vivant: do kamo, l'humain vrai, à la différence de l'animal et du dieu, dépasse l'image physique et la référence au corps. Depuis la biologie, le vivant est devenu une partie du corps par son organisation et ses fonctions, facilitant ainsi la naturalisation et l'identification du corps. Pour le Canaque, le corps supporte le vivant, tandis que pour nous, le vivant contient le corps.

Le chercheur apporte le corps, sinon son corps, dans les progrès mesurables depuis l'observation par l'ethnologue des Canaques :

Voulant mesurer le progrès accompli chez les Canaques que j'avais instruits de longues années, je dis au vieux Boesoou, ancien sculpteur et détenteur des discours de pilous :

- «En somme, c'est la notion d'esprit que nous avons portée dans votre pensée ?»

Et lui d'objecter :

«L'esprit? Bah! Vous ne nous avez pas apporté l'esprit. Nous savions déjà

l'existence de l'esprit. Nous procédions selon l'esprit. Mais ce que vous nous avez apporté, c'est le corps. »

(Leenhardt $1942:$ :114)

Leenhardt cherche à qualifier les effets de l'observation sur l'individuation, car toute observation produit une évasion du domaine socio-mythique initial, mais l'ethnologue se trompe, en proposant l'esprit, comme progrès accompli. Leenhardt n'a donc pas conscience de son corps et du corps comme condition d'une individuation, projetant l'esprit comme un facteur de dégagement et d'individuation pure de la personne. Leenhardt écrit, juste avant de révéler la réponse de Boessou, que la personne mélanésienne "est participative, elle est une réalité humaine que le Canaque a pressentie en la désignant au travers du pronom, "le lui", et en lui donnant le corps comme support. Mais il n'a pu aller plus avant » (Leenhardt 1942 : 114).

La surprise et l'écart entre les deux moments de l'analyse révèlent la différence entre ce qu'apporte l'ethnologue comme progrès et l'impossibilité pour le mythe de " contenir ce qui échappe aux sens et qui est précisément humain " (Leenhardt 1942 : 114), le corps. Mais le Canaque en est conscient, puisque, dans sa réponse, c'est le corps, plutôt que l'esprit, qu'il identifie comme ce qui constitue la nouveauté introduite par rapport à la structure mythique, comme s'il témoignait déjà de ne plus y être maintenu tout à fait. Ce qui est une preuve de l'individuation, doublée d'une nomination et d'une conscientisation de l'apport étranger de l'ethnologue. Ainsi le chercheur peut apporter le corps dans une culture qui ne le possédait pas, et un corps fondé sur la personne et l'individuation qui sont la preuve d'une «délimitation ferme " et d'un "contour nouveau » (Leenhardt 1942 : 115).

Le corps produit, par son introduction comme "circonscription de l'être physique ", une circonscription épistémologique, car il avait jusque-là "part à toutes les 
participations mythiques » mais «n'était qu'un soutien » sans « existence propre » ni "nom spécifique ». L'objectivation de l'être physique dans le corps est rendue possible par l'achèvement de sa circonscription.

L'enjeu épistémologique est de faire le lien entre l'observation sociologique de Durkheim et l'observation ethnologique de Leenhardt, Durkheim produisant le concept d'individuation au moment même où, en 1912, Leenhardt le produit dans la société mélanésienne :

Vers l'époque même où le vieux Boesoou résumait ainsi sa propre expérience, et en généralisait si clairement la conclusion, Durkheim, par les voies sociologiques et celles de sa profonde vision, aboutissait à un résultat pareil et notait d'intuition : «Il faut un facteur d'individuation. C'est le corps qui joue ce rôle.» (Leenhardt 1942 : 115 - Leenhardt cite ici Durkheim $1912:$ 386)

Le corps jouerait donc un rôle dans la formation de la personne dans toute société, ce qui occulterait la responsabilité du chercheur à l'égard de l'introduction du corps dans la société mélanésienne. Ce qui est certain c'est que cet apport produit l'individuation comme si le domaine socio-mythique enfermait et cantonnait la personne dans « un personnage sans rôle » (Leenhardt $1952: 115)$.

L'affirmation "j'ai un corps », et non pas «je suis un corps ", apparait par une double opération du Canaque qui «aperçoit l'indépendance de son existence corporelle et enrichit sa langue en versant dans le mot ancien le contenu du concept nouveau: corps" (Leenhardt 1942: 115). Ainsi le support du kamo est un karo "qui exprime l'idée d'un soutien formant un ensemble avec le soutenu» (Leenhardt 1942: 103); s'appuyant sur l'analyse de Marcel Mauss (voir Mauss 1938), il explique qu'un homme est seulement « une silhouette que l'on a devant soi, et l'on ne sait quel personnage elle recouvre» (Leenhardt 1942: 103), comme n'importe quel autre support, «manche de hache, pied de table, masse d'eau du fleuve, creux d'un trou, essaim, troupe de guerre » (Leenhardt 1942 : 103). L'identification du support « lui donne sa place dans le monde sensible et son rôle l'intègre dans la société » (Leenhardt 1942 : 104). En ne discriminant pas entre le corps et le rôle, cette juxtaposition laisse la différence comme "virtualité personnelle » (Leenhardt $1942: 104)$ lors de «l'action et réaction du corps et rôle » qui «modifient le personnage». Le double aspect du kamo superpose le corps et le rôle sans favoriser une séparation identifiante pour le moi. Le corps n'est jamais rencontré comme tel, "mais bien [comme] un mâle, une femme, ou un personnage en son rôle » (Leenhardt $1942: 105$ ).

31 Leenhardt est bien conscient de la projection de son corps d'Occidental, dans le cas de la mort et du statut du cadavre : « On serait fondé à croire que le ko est le principe vital du corps. Mais ce serait juger là avec une mentalité d'Europe, qu'elle soit dualiste et oppose corps et esprit, ou qu'elle procède simplement du principe de contradiction, et oppose corps et esprit. » (Leenhardt 1942 : 108, note 2) En indiquant que ko karo, esprit du corps, est une expression « incompréhensible au Canaque », l'ethnologue reconnaît que «tant se complique l'intelligence de la pensée primitive lorsque nous voulons la plier au moule de notre langage » (Leenhardt $1942: 109)$ !

La colonisation modifie le sens des mots en décontextualisant la signification traditionnelle des relations, comme la femme enceinte dont le mari n'a que l'usufruit de la vie qu'elle porte, la femme étant la propriété de son propre clan ${ }^{5}$. La propriété corporelle n'a pas de sens, car ce qui reste personnel est déjà engagé dans l'alliance avec le clan (Leenhardt 1942: 129). L'opposition entre le langage ethnologique et le 
langage rationnel ne résout pas la question de diffusion de la notion de personne ; dans le premier cas la personne est à l'état diffus et dans le second l'objectivation (Leenhardt 1942 : 136) réduit la personne.

La méthode établie par Leenhardt démontre combien le corps du chercheur exporte son langage, sa conceptualisation et son processus d'identification dans une culture étrangère à celle du chercheur lui-même. En distinguant ce qui provient de lui de ce qui appartient à la culture, on fait du corps une catégorie repérable dans sa projection épistémologique, mais aussi dans sa colonisation ontologique: le corps crée la conscience de la catégorie de personne qui, jusqu'à la venue de l'homme blanc, n'était pas unifiée.

Maurice Godelier et Michel Panoff sont bien conscients de ce principe découvert par Leenhardt lorsqu'ils estiment que la question est de savoir « jusqu'à quel point le corps fait l'identité d'un être humain et pour combien de temps, puisqu'il est destiné à mourir » (Godelier et Panoff 1992 : XII). Le corps ne suffit pas pour produire des êtres humains, telle est la thèse défendue : «Il faut ajouter une ou plusieurs choses au corps humain pour qu'il soit le corps d'un être humain» (Godelier et Panoff 1992 : XIII). Ainsi le corps ne suffit pas à lui tout seul, et encore moins le corps du chercheur, pour instaurer une dualité en tout être humain sans obligatoirement y projeter notre dualisme occidental. Le modèle corps et âme fait croire en une unité de principe dont l'association suffirait pour constituer l'identité humaine : « La corporéité humaine n'est jamais pleinement garante de l'humanité d'un corps » (Godelier et Panoff 1992 : xv).

Ainsi « pour comprendre le corps il faut combiner deux approches. Analyser la manière dont une société se représente la nature et l'origine des substances, des organes et des fonctions qui composent un corps, mais en même temps analyser ce qui est supposé exister dans ce corps derrière l'enveloppe de la peau, qui l'anime et surtout en contrôle les actes » (id. : XVIII). Le corps ne provient pas de lui-même, il est produit par les autres - d'autres humains, des dieux, des esprits, ce qui relativise la responsabilité individuelle de ce qui serait le propre, sinon la propriété, du corps. La subordination politique, économique et religieuse «se marque dans le corps et c'est pour cela que le contrôle des corps et leur appropriation sont un enjeu dans toutes les sociétés » (id. : XIX).

Le sang, le sperme, le lait «sont non seulement polysémiques, mais surchargés de sens, parce qu'ils sont en quelque sorte "surdéterminés" par un certain nombre de fonctions sociales » (id. : xx). C'est "dans le corps » (id. : xxI), par le moyen des rites de passage, des nourritures obligées ou interdites, des parures, que se construit «la supériorité ou la subordination des individus".

\section{L'hybrigraphèse érographique}

37 En remplaçant le pénis par le gode, la société contra-sexuelle de Beatriz Preciado (2000) libérerait définitivement la femme de la nature et du pouvoir phallocentrique du mâle. L'utérus artificiel (voir Atlan 2005), après la contraception, l'avortement, la fécondation in vitro et le clonage, confirmerait le progrès en abandonnant entièrement le corps de la femme comme réceptacle naturel. La pornographie féministe d'Ovidie (voir Ovidie 2002) renverserait la domination masculine en inversant les rôles et en détournant les codes de la jouissance sociale - ce dont doutent les moralistes de la sexualité (voir Marzano 2005). Le kamasutra moderne devrait trouver dans les sex toys 
(voir Ploton 2004) les règles d'un jeu conventionnel. Le fétichisme, l'échangisme et le $\mathrm{SM}^{6}$ habillent désormais le désir de l'autre que l'on ne pourrait toucher qu'à travers des dispositifs de contrat et de commerce. Le blog socio-cul dressant des statistiques de performances et des fiches sur le vécu sexuel ${ }^{7}$, les réseaux et les pornogames sur Internet $^{8}$ achèvent la dématérialisation du corps de la femme.

Déjà Peter Greenaway, avec le système Pillow Book, écrivait sur la peau de treize hommes, le corps devenant le pinceau plutôt que le papier. Dans 100 Allegories to Represent the World, l'artiste définissait un cliché body painting en confondant le corps dans la toile qui l'enrobe. Le body painting hybride la peau, le sexe, les seins, les fesses à la manière de la Gestalt psychologie: le détournement de la forme naturelle par le surlignage coloriste met en relief un corps virtuel. Retournant le maquillage, le masque de couleur sur tout le corps l'extrasocialise en l'offrant moins en spectacle ou en pâture à la perception désirante qu'il ne l'inscrit au sein même de la société.

39 La trace sur la peau a souvent été comprise, comme par David Le Breton, Catherine Rioult et Marion Haza, comme une écriture du sujet souffrant : le tatouage, l'incision ou la scarification participent de la construction identitaire là où la peinture de Karl Lakolak l'incarne (voir Lakolak 2007). À la différence du cliché érographique, le cliché pornographique, est, littéralement, un cliché : pour qui surfe sur youporn.com, aucune inventivité n'est visible, la mise en scène de postures standard est organisée en fonction de la webcam ou de la vidéo-DVD: sodomie, gorge profonde et autres vibromassages ne font qu'épuiser le sujet dans l'orgasme sans montrer la chair vécue.

Dans le cliché érographique ${ }^{9}$ ce n'est plus seulement l'amant qui s'écrit sur la peau de l'autre, ni l'aimant qui attire le designer. La chair s'exsude en émergeant de la peau. La transparence produite par l'ex-carnation: plusieurs couches d'incarnat du crâne à l'exoderme révèlent, au sens photographique, la pluralité biohistorique du sujet s'incarnant. Les multicouches de couleurs sont celles, esthésiologiques, qui, de la chair intime jusqu'à la peau intersubjectivante, vont bien nous révéler l'intimité vécue. Là où la radiographie découvre in vivo le squelette et l'activité neurocognitive, l'érographie dévoile les marqueurs somatiques affectifs de la chair vécue : les couches de couleurs sont autant de tomographies des affects in vivo, affects du sujet et affects de l'artiste, sinon du spectateur. La projection de couleur indique les variations et l'intensité des affections ressenties.

41 Le sexe masculin est maculé moins de sang que de sens, la couleur épaississant le pénis pour rendre désirable sa carnation symbolique. La couleur rend la matière désirable, là où le teint naturel de la peau ne suffit plus à nourrir le désir: sans symbolisme qui établirait une correspondance entre affect et couleur, la couleur transmue et transgenre la nudité et le sexe. La couleur et les motifs pourraient homosexuer les corps alors qu'elles dégenrent les peaux pour les rendre désirables. Chaque couleur est érotographique.

Yves Klein n'aura pas suffi à rendre la peau colorée désirable. Le métissage des couleurs détisse la peau naturelle pour inventer l'horizon arc-en-ciel du mélange. L'érographie hybride le modèle et l'artiste en les produisant dans un devenir réciproque. La peau nous retourne par les couleurs, leurs pigments et leurs piments. Entre le modèle et l'artiste, la peau est comme une interface. Surface, support, fond, feuille, l'écritoire tactile rend le texte du ventre même de la chair. Les mots se perdent dans la chair parce qu'ils en proviennent; ils ne la traversent pas, ils s'inversent en passant de l'opaque à la transparence. Le sens se change dans le signe coloré, la phrase 
indéfinissable du vécu affectif interdit toute clôture de la représentation par l'intense présence de l'ouverture.

En passant à travers sans être rejeté au dehors du corps, le cliché érographie sans délimiter et sans parvenir à border le désir et le sens. Le rouge est à la fois le sang, le sexe et la mort, nous conduisant de l'un dans l'autre sans que nous y abandonnions jamais notre regard aussitôt engagé dans la couche suivante, l'espace corporel proche. La proximité des corps trouve dans les fentes de couleurs mieux que les orifices naturels autour desquels le corps s'enroule depuis sa formation : la bouche anus est un puits sans fond face à cette multitude de réseaux transversant le sens par l'obliquité des couches de couleurs.

\section{L'hybrigraphèse cyborgique}

L'apprentissage des techniques du corps et des objets corporels s'inscrit dans une synthèse corporelle par laquelle, en faisant corps avec la technique par l'objet, on arrive à "l'incorporation de la dynamique de l'objet à titre de prothèse dans une conduite motrice » (Warnier 1999: 10). L'objet n'est donc pas extérieur au corps, mais sa dynamique définit une prise intériorisée "par tous les points de contact et de perception entre la chose et le sujet : doigts, mains, pieds, siège, dos, toucher, ouïe, vue, perception gravitationnelle par l'oreille interne, proprioception neuro-musculaire " (Warnier 1999: 11).

La mémorisation des conduites motrices est l'objet d'une "praxéologie » ou science de l'action motrice, comme le montrent les travaux de Pierre Parlebas (voir Parlebas 1981). Il n'y a pas d'article corps dans le lexique car il s'agit d'éviter « les deux versants solidaires de l'épistémologie: le versant de l'épistémologie interne (critique des concepts et de la méthodologie adoptés) et celui de l'épistémologie externe (analyse du contexte socio-institutionnel) » (Parlebas 1981: XXII). Organisatrice signifiante du comportement moteur, la conduite motrice n'est observable qu'indirectement et après coup, car les mécanismes de préaction, les interactions motrices et le réseau de communication n'isolent pas l'objet de la situation de jeu.

Le cognitivisme établit bien l'engagement du corps dans l'action à partir d'une connaissance non discursive de la relation du sujet à l'objet (voir Conein et al. 1993). L'action sert alors d'intermédiaire pour engager le corps selon la fonction technique de l'objet. La culture matérielle n'en reste pas à la notion insuffisamment élaborée de corps qui ne parvient pas à distinguer clairement entre techniques du corps et techniques instrumentales.

Face aux défaillances et aux dysfonctionnements de notre corps vieillissant et malade, nous incorporons dans et sur notre corps des objets, des techniques et des habitus qui modifient notre schéma, notre image et notre vécu corporels. Ces compensations deviennent des modifications intégrées dans nos modes d'existence, d'action et de relation au monde, aux autres et à nous-mêmes.

La technique n'est plus seulement à comprendre comme une extériorisation du geste dans le monde, mais aussi comme une internalisation du monde dans le corps. Plus que des techniques du corps, cette hybridation du corps le recompose en un mélange de fonctions induites par des techniques, outils, prothèses, implants... et les compensations motrices, perceptives et relationnelles de notre corps. L'hybridation peut remédier à un manque (Maillet 2007 : 17) en le compensant, mais en modifiant par 
la plasticité adaptative du corps nos schèmes de perception et d'action. L'hybridation implique un monitoring permanent (voir Dittmar et al. 2007), renforçant à la fois le biopouvoir et l'autosanté selon le point de vue politique.

L'interaction entre la technique et le corps produit l'hybride. L'hybride n'est pas seulement le cyborg de Donna Haraway dont le mythe a pour fonction politique de servir « une histoire de frontières transgressées, de fusions redoutables et de possibilités dangereuses » (Haraway $1991: 275)$. L'hybride est le résultat pragmatique et sensori-moteur: comme queer technologique, l'hybride est une culture populaire (Lauretis $1990: 84$ ) de pratiques corporelles des objets (voir Preciado 2000) et de leurs conséquences (voir Maines 1999) sur le vécu corporel. La dénaturalisation et la dégenderisation (voir Butler 2005) doivent s'accomplir en assumant positivement l'hybridation de notre corps plutôt qu'une dépendance, une aliénation et une instrumentalisation; l'hybridation ne dit pas adieu au corps et n'aspire pas au posthumanisme.

Devenir hybride dépasse les oppositions traditionnelles en les comprenant dans l'unité dynamique du corps : par son recalibrage le corps se réorganise en compensant les effets de l'hybridation (Malabou 2006: 540). La régénération passe à la fois par la culture in vitro des cellules-souches (voir Donarin 2006) et autres produits biotechnologiques et par l'implémentation de prothèses (voir Alegria et al. 2007). L'hybridation, entre fantasme et expérimentation, est une propriété naturelle de reproduction avant que la science horticole ne la découvre afin de constituer une mise en culture (voir Poliakov 1975). L'hybride est présent dans la chimère biologique et métaphorique comme dans le corps double de l'hermaphrodite : Michel Serres voit en la chimère Sarrasine de Balzac, celle qui « croise le lien commun aux arts comme nos corps communs, chimériques, croisent les liens qui les unifient »(Serres $1987: 118$ ).

51 L'hybridation selon Henry Dougan est bivalente: si l'hybridation remplace la pureté par le mélange, la stabilité par un processus ouvert, la différence moi/autre par l'égalité humaine, « les processus d'hybridation perturbent les frontières fixées, ceux-ci peuvent provoquer des réactions brutales visant à renforcer des marqueurs essentialistes » (Dougan 2004 : 39). Contre les valeurs essentialistes de pureté et de stabilité, l'hybridation pourrait représenter la défense d'une éthique du métissage et de la tolérance ; mais, comme le gender et le queer, le renversement des normes convenues produit des crises identitaires du corps social en raison du brouillage des marqueurs d'identité. Le nationalisme, le racisme, le sectarisme et le terrorisme sont les contreparties sociopolitiques de la venue de l'hybridation comme alternative : «Une hybridation plus intense peut provoquer des réactions plus violentes et plus discriminatoires » (Dougan $2004: 38$ ).

L'anthropobiologie culturelle de Dougan n'est pas eugénique ; c'est celle de « la grande raison d'un corps multiple, transgénique, impur, hybride, artificiel, prothésé et créole » (Lafargue 2007: 64). L'hybridité, devenue un lien entre politiques culturelles et pratiques artistiques (voir la journée d'études Jeunes Chercheurs Politiques culturelles 2005), fonde désormais la relation sociale sur la tolérance et le métissage. En incorporant, jusque dans la matière biologique, la part de l'autre, le sujet devient mixte : prendre le parti de la chair, c'est vivre la matière altérée par l'interaction environnementale. Au lieu que je devienne un autre, les autres deviennent une partie de moi en modifiant le fonctionnement et la subjectivité du corps. 

notre corps, constitue notre identité dans bien des actes de notre vie quotidienne. Les transformations sont visibles. Les objets techniques transforment notre corps en le rendant dépendant. La vie hybridée nous oblige à repenser les distributions sociales de genre, les rapports que nous entretenons avec les machines et la santé de notre corps. La technique n'est plus à l'extérieur de nos corps, mais la peau sert désormais d'interface entre le dedans et le dehors : le son, la vue, le toucher, la mesure, le rythme, l'alimentation, la respiration, etc., sont désormais relayés par des I.peauds (au sens de I.pod). Accepter de vivre greffés, implantés, branchés définit une interaction positive entre la peau et le monde : la peau n'est plus une surface biologique ou un miroir de notre apparence; elle devient un mode d'être par l'insertion dans ces pores mêmes des canaux de l'information, de la télémesure et de l'expression des émotions. La machine est une part de notre peau, plutôt que la peau ne devient machinale.

\section{Conclusion}

Se dresse pour le $\mathrm{xxI}^{\mathrm{e}}$ siècle une nouvelle perspective où l'âme peut s'incarner directement dans le corps par une hybrigraphèse intense de la composition matérielle et de la surface du corps lui-même. Le corps est désormais étendu de l'intérieur de son âme à l'extérieur de ses prothèses en rendant interchangeables ses parties avec des kits bioartificiels. Le corps n'est plus la substance étendue du dualisme cartésien mais une matière à formes qui sera biosubjectivée selon l'intention hybridante d'un sujet.

Le sujet modifié est en même temps le sujet modificateur, à la fois effet et cause de son propre corps. Se démarquant de ses origines naturelles et refusant de subir le déterminisme génétique, l'hybride, à l'inverse de l'utopiste spiritualiste, est un matérialiste convaincu : plus qu'un corps à son image, il établit une continuité moniste dans le corps lui-même en allant du corps pensant au corps modifié. Une variation d'effets est induite par l'insertion des biotechnologies dans la chair même du corps.

Le temps est venu de renoncer au modèle mécanique d'un programme génétique qu'il suffirait de répliquer pour reproduire des êtres identiques et des organismes génétiquement modifiés. François Dagognet précise: "c'est pourquoi, à tenter de le remanier ou de le subvertir, nous n'obtiendrons pas un "corps redressé" ou "reconstitué", - la guérison, le prétendu retour à ce qui était perdu (restitutio ad integrum)-, mais un "corps probablement insolite" sinon inapte, plus fragile normalisé peut-être mais nullement normatif. » (Dagognet 2001 : 333)

Le style incarné personnalise le corps à l'envers de la mode récupératrice de nos libertés. La différence entre individualisation indéfinie du corps incarné et hédonisme individualiste se fonde sur les multi-partenariats sexuels, et l'attachement au corps originel et naturel de la tradition conservatrice ne rend plus compte des pratiques d'hybrigraphèse de l'existence; l'être incarné produit un corps mélangé car le sujet doit composer ses formes selon les mutabilités de la matière. Ces érotisations et ces esthétisations du corps transforment le rapport du sujet à son corps et aux autres corps par le mélangisme et le métissage. 


\section{BIBLIOGRAPHIE}

Alegria J., Deltenre, P., Leybaert, J. et Serniclaes, W., 2007, Surdité et Langage. Prothèses, LPC et implant cochléaire, PUV.

Andrieu, B., 1993, Le Corps dispersé. Une histoire du corps au XXe siècle, Paris, L'Harmattan.

- 2008, Devenir hybride, préface de Stelarc, Nancy, Presses Universitaires de Nancy.

Artières Ph., 1998, Clinique de l'écriture. Une histoire du regard médical sur l'écriture, Paris, Les Empêcheurs de penser en rond.

Atlan, H., 2005, L’Utérus artificiel, Paris, Le Seuil.

Benatouil, T., 2003, « Foucault stoïcien?», dans F. Gros et C. Lévy (dir.), Foucault et la Philosophie antique, Paris, Kimé.

Butler J., 2005 [1990], Trouble dans le genre. Pour un féministe de la subversion, Cynthia Kraus (trad.), Paris, La Découverte.

Conein, B., Dodier, N., et Thevenot L. (dir.), 1993, Les Objets dans l'action. De la maison au laboratoire, Paris, Éditions de la Maison des Sciences de l'Homme.

Courtoux, S., 2006, Action-Writing (manuel), Cognac, Le temps qu'il fait.

Dagognet, F., 2001, « Le Retour de quelques illusions », dans L. Sfez (dir.), L'Utopie de la santé parfaite, colloque de Cerisy, 1998, Paris, PUF.

Denan, P., 2004, Libre 3, Paris, M19.

Dittmar, A., Gehin, C, McAdams, C. et al., 2007, « Microcapteurs, vêtements, habitats intelligents pour le monitoring permanent : impact société et éthique ", dans M.-A. Grimaud, C. Hervé, M.S. Jean et al. (dir.), La Nanomédecine. Enjeux éthiques, juridiques et normatifs, Paris, Dalloz.

Dougan, H., 2004, « Hybridation, promesses et limites », Bulletin du Codesria, $\mathrm{n}^{\circ} 1$ \& 2, p. 36-42, ici p. 39.

Durkheim, É., 1985 [1912], Les Formes élémentaires de la vie religieuse, Paris, PUF.

Farge, A., 2003, Le Bracelet de parchemin. L'écrit sur soi au XVIII siècle, Paris, Bayard.

-, 2007, Effusion et Tourment, le récit des corps. Histoire du peuple au XVII siècle, Paris, Odile Jacob.

Fassin, D. et Memmi, D., 2004, Le Gouvernement des corps, Paris, Éditions de l'EHESS.

Foucault, M., 1983, Le Gouvernement de soi et des autres, Paris, Gallimard-Le Seuil, 2008.

-, 1984a, « Entretien avec H. L. Dreyfus et P. Rabinow [1983] », dans H. L. Dreyfus et P. Rabinow (éd.), Michel Foucault. Un parcours philosophique, Paris, Gallimard.

-, 1984b, Le Souci de soi, t. 3, Histoire de la sexualité, Paris, Gallimard.

_, 2001, L’Herméneutique du sujet [cours 1981-1982], Paris, Gallimard-Le Seuil.

Godelier, M., et Panoff, M., 1992, «Introduction », dans M. Godelier et M. Panoff (dir.), La Production du corps, Approches anthropologiques et historiques, Paris, Éditions des Archives contemporaines.

Haraway, D., 2008 [1991], Des singes, des cyborgs et des femmes. La réinvention de la nature, O. Bonis (trad.), préface M.-H. Bourcier, Paris, Jacqueline Chambon. 
Jardine, A., 1991 [1985], Gynesis. Configurations of Woman and Modernity, Ithaca, Cornell University Press. [Trad. fr, Gynésis. Configurations de la femme et de la modernité, Paris, PUF.]

Kraus, C., 2005, “"Avarice épistémique” et économie de la connaissance : le pas rien du constructionnisme social », dans H. Rouch, E. Dorlin et S. D. Fougeyrollas-Schwebel (dir.), Le Corps, entre sexe et genre, Paris, L'Harmattan, coll. « Bibliothèque du féminisme », p. 39-59.

Lafargue B., 2005, «Dans l'œil de la bête », dans B. Lafargue (dir.), Animaux d'artistes, $\mathrm{n}^{\circ} 8$ de Figures de l'art.

Lakolak, K., 2007, Sarabande pour un corps, Paris, Everland.

Lakolak, K., 2008, Érographies d'incorporelles, Paris, Everland.

Lauretis, T. de., 2007 [1990], Théorie queer et cultures populaires. De Foucault à Cronemberg, M.H. Bourcier (trad.), Paris, La Dispute.

Le Douarin, N., 2007, Les Cellules souches, porteuses d'immortalité, Paris, Odile Jacob, p. 19-24.

Leenhardt, M., 1942, « “Le Corps”. La personne mélanésienne ", Annuaire de l'École Pratique des Hautes Études, section des sciences religieuses, 1941-1942, Melun, p. 5-36, repris dans La Structure de la personne en Mélanésie, Milan, Maussiana, 1970, p. 114.

-, 1971 [1947], « La notion de corps », Do kamo, Paris, Gallimard, coll. « Tel », 1971.

Maillet, A., 2007, Prothèses lunatiques. Les lunettes, de la science aux fantasmes, Kargo, Éditions Amsterdam.

Maines, R., 1999, Technology of Orgasm: "Hysteria", the Vibrator and Women's Sexual Satisfaction, Baltimore, The John Hopkins University Press.

Malabou, C, 2006, «Les Régénérés : cellules-souches, thérapie génique, clonage », dans Mutants, $\mathrm{n}^{\circ}$ 709-710 de Critique.

Marguerite, V., 1927, Ton corps est à toi !, Paris, Flammarion.

Martin, E., 1987a, The Woman in the Body, Boston, Beacon Press.

-, 1987b, « Medical Metaphors of Women’s Bodies », dans Martin 1987a, p. 27-67.

Marzano, M., 2007, « Le corps est un adversaire », Le Monde, 21-22 avril.

Mauss, M., 1938, « Une catégorie de l'esprit humain, la notion de personne, celle de "moi" ", Royal Anthropological Institute.

Marzano, M., 2005, Entretien avec Ovidie. Le corps acteur, Paris, Autrement.

Meidani, A., 2006, Les Fabriques du corps, Toulouse, Presses Universitaires du Mirail.

Oudshoor, N., 2000, « Au sujet du corps, des techniques et des femmes », dans D. Gardey et I. Lowy (dir.), L'Invention du naturel, Paris.

,- 1990 , « On the Making of Sex Hormones: Research Materials and the Production of Knowledge », Social Studies of Sciences.

Ovidie, 2002, Porno manifesto, Paris, Flammarion.

Parlebas P., 1981, Lexique commenté en science de l'action motrice, Paris, INSEP.

Ploton, Fr., 2004, Sex Toys, Paris, Tana. 
Poliakov, L., 1975, « Le Langage des êtres hybrides et la hiérarchie des races aux XVIII ${ }^{\mathrm{e}}$ et XIX ${ }^{\mathrm{e}}$ siècles ", dans L. Poliakov (dir.), Hommes et Bêtes. Entretiens sur le racisme, actes du colloque tenu du 12 au 15 mai 1973, Paris, La Haye et Mouton, Éditions de l'EHESS, p. 171-185.

Politiques culturelles face à l'hybridation des pratiques artistiques (Les), 2005, journée d'étude Jeunes Chercheurs ED/MSH Alpes, 27 mai 2005.

Preciado B., 2000, Manifeste contra-sexuel, M.-H. Bourcier (trad.), Paris, Balland.

Prochasson, Chr., 2008, « L'histoire à la première personne », L'Empire des émotions. Les Historiens dans la mêlée, Paris, Démopolis.

Sammoun, M., 2004, Tendance SM : Essais sur la représentation sadomasochiste, Paris, La Musardine.

Serres, M., 1987, L'Hermaphrodite. Sarrazine sculpteur, Paris, Flammarion.

Soulié, B. et Corneloup, J., 2007, Sociologie de l'engagement corporel. Risques sportifs et pratiques " extrêmes » dans la société contemporaine, Paris, Armand Colin.

Streff, J., 2005, Traité du fétichisme à l'usage des jeunes générations, Paris, Denoël.

Travaillot, Y., 1998, Sociologie des pratiques d'entretien du corps. L'évolution de l'attention portée au corps depuis 1960, Paris, PUF.

Varela, F., Thompson, E. et Rosch, E., 1993, «L'énaction : cognition incarnée », dans L'Inscription corporelle de l'esprit. Sciences cognitives et expérience humaine, Paris, Le Seuil.

Warnier, J.-P., 1999, Construire la culture matérielle, Paris, PUF.

Weltzer-Lang, D., 2005, La Planète échangiste. Les sexualités collectives en France, Paris, Payot.

\section{NOTES}

1. "Although women resist specific medical procedures such as cesarean section or anesthesia during delivery, they seem unable to resist the underlying assumptions behind these procedures: that self and body are separate, that contractions are involuntary, that birth is production. " (Martin 1987 : 89 ; sauf indication contraire, les traductions sont de Pierre Zoberman.)

2. "The laboratory not only reflects gender bias in society, it is the very place where gender is constructed and metamorphosed» (Oudshoor $1990: 26$ ).

3. «Disons que la substance éthique des Grecs était les aphrodisia; le mode d'assujettissement était un choix politico-esthétique. La forme d'ascèse était la technè utilisée et où l'on trouve par exemple la technè du corps, ou cette économie des lois par lesquelles on définissait son rôle de mari, ou encore cet érotisme comme forme d'ascétisme envers soi dans l'amour des garçons, etc. ; et puis la téléologie était la maîtrise de soi.» (Foucault 1984a : 335-336)

4. Selon l'expression de Sylvain Courtoux: «L'Assomption dernière du corps signifiant est politique : continuer sa propre usure en usant des mots étouffer de n'avoir dans sa bouche que la langue maternelle dit-elle (voilà tout ce que dit l'écho) ventre même humide humilié face à face ô pénis engorgés en-foutrés trop de merde trop de derme novlangués mater-n(i)és mon sperme chié dans le chiasse-misme matern-fille... » (Courtoux $2006: 12$ ).

5. Leenhardt se réfère ici au livre de Victor Marguerite (1866-1942), Ton corps est à toi ! (1927).

6. Voir Weltzer-Lang 2005, Streff 2005 et Sammoun 2004.

7. Voir le site http://jeniquecestmythique.free.fr/ (consulté en novembre 2006).

8. Voir le site http://www.virtuallyjenna.com/. 
9. Je renvoie ici à Lakolak 2008 où figure une version antérieure de ces réflexions sur ses érographies, sous le titre "Le Cliché érographique» (p. 52-55). (Voir aussi les œuvres de Karl Lakolak reproduites dans ce volume [NDLR].)

\section{RÉSUMÉS}

De l'idiosyncrasie de Nietzsche au biographème de Barthes, l'inscription de son corps dans le texte devrait être la condition d'une lecture épistémologique du corps du chercheur dans son travail. Cet article décrit la façon dont le corps du chercheur est intégré à sa recherche par le biais de son genre ou de sa langue. Cet article utilise l'épistémologie du corps pour essayer d'inventorier ce processus théorique du mentir vrai, processus qui est plus ou moins conscient chez le chercheur.

From Niezsche's idiosyncrasy to Barthes's biographeme, inscribing one's body into the text should be the condition for an epistemological reading of the researcher's body in his/her work. This article describes the way in which the researcher's body is brought into his/her research through and by either his/her gender or his/her language. The present article uses the epistemology of the body to try to map out this theoretical process of lying truthfully, a process which is more or less conscious in the researcher.

INDEX

Mots-clés : biopouvoir, cyborg, épistémologie du corps, hybridation, écriture

Keywords : biopower, cyborg, eptistemology of the body, hybridization, writing

\section{AUTEUR}

\section{BERNARD ANDRIEU}

VHP-Nancy Université, ACCORPS \& LHSP UMR 7117 \& GDR 2322 CNRS 